\title{
STANDARDISED FEEDING STRATEGIES IN TERTIARY NEONATAL UNITS: A SURVEY OF CURRENT PRACTICES IN THE UNITED KINGDOM
}

\author{
H.K. Muniraman ${ }^{1}$, R. Alanoor ${ }^{2}$, R. Roy ${ }^{2}$ \\ ${ }^{I}$ Neonatalogy, Norfolk and Norwich Hospital, ${ }^{2}$ Neonatal Intensive Care Unit, Norfolk \& Norwich University \\ Hospitals NHS Trust, Norwich, UK
}

Background: Standardised feeding strategies have shown to minimise variations in enteral feeding practices which reduces the risk of necrotising enterocolitis (NEC). The pathogenesis of NEC is multifactorial and over $90 \%$ of the infants develop this condition after being fed.

Methods: Telephone survey of all 40 tertiary neonatal units in the United Kingdom enquiring their enteral feeding practices in premature infants.

Results: 26 (65\%) units followed a standardised enteral feeding guideline, of which 6 units had seen a reduction in incidence of NEC following implementaion of the guideline. Expressed maternal breast milk was the preferred milk in all units. $30(75 \%)$ units would consider using donor breast milk as second choice. 38 (95\%) units used trophic feeds to promote gut maturation and for early establishment of enteral feeds. $25 \%$ units would delay commencing enteral feeds if umbilical catheters were in situ. $98 \%$ units would delay commencing enteral feeds varying between 1 to 5 days in case of absent or reversed end diastolic umbilical blood flow.

$27(68 \%)$ units defined gastric residual volume (GRV) $>50 \%$ of previous 4 hour feeds as feed intolerance whilst it was $>25 \%$ in $7(17 \%)$ of units. Bile stained aspirates and abdominal wall discoloration was attributed to feed intolerance or suspected NEC in all units.

Conclusion: Enteral feeding practices and assessment of feed intolerance varied in majority of the tertiary neonatal units in UK. Uniform guidelines for feeding intolerance will reduce the variation in practice as these remains largely subjective rather than evidence based. 